\title{
Análisis de las relaciones entre los ciclos económicos internacionales
}

\author{
Sonia de Lucas Santos \\ Departamento de Economía Aplicada (Udi de estadística) \\ U. Autónoma de Madrid \\ Campus de Cantoblanco, 28049 - Madrid \\ Email: sonia.delucas@uam.es
}

\section{Inmaculada Álvarez Ayuso}

Departamento de Análisis Económico

Teoría Ec. e Historia Ec., U. Autónoma Madrid

Campus de Cantoblanco, 28049 - Madrid

Email: Inmaculada.alvarez@uam.es

\section{Ma Jesús Delgado Rodríguez}

Departamento de Economía Aplicada II

Universidad Rey Juan Carlos

Campus de Vicálvaro, 28032 Madrid

Email: mariajesus.delgado@urjc.es

\section{INTRODUCCIÓN}

En años recientes los estudios sobre ciclos económicos a nivel mundial, han recobrado importancia debido a las sucesivas contracciones y crisis económicas que se han producido en el contexto internacional y que han producido efectos muy diferentes en los países y regiones del mundo. A esto se une el interés que suscitan la variedad en las técnicas para extraer el ciclo económico y la disponibilidad de estadísticas que permiten ampliar el número de países estudiados. Los trabajos en esta línea se han centrado fundamentalmente en analizar la sincroni- 
zación entre los ciclos con estudios de la Unión Europea (EU), para ver si ante medidas de política estabilizadoras, todos los países miembros reaccionan igual y sufren las mismas consecuencias; destacando los trabajos de Harding y Pagan (2002), Artis, Marcellino y Proetti (2004), Camacho, Pérez-Quiros y Saiz (2005, 2006) y Krolzing y Toro, 2005; o bien, en los países del G7 mostrando una mayor estabilidad del ciclo económico de estos países en las últimas dos décadas (Stock y Watson, 2003), y en la OCDE (Cancelo y Uriz, 2001; Cotis y Copel, 2005). Sin embargo, son pocos los trabajos que tratan de evaluar el ciclo a nivel mundial como el informe del Fondo Monetario Internacional (FMI) de octubre de 2007, que recoge un análisis exhaustivo de los cambios en la dinámica de un ciclo económico global para 133 países.

En este artículo se presentan las principales herramientas para el análisis de los ciclos que permitirán obtener una visión general del ciclo internacional, en términos del ciclo económico del PIB per cápita, siendo el indicador de actividad económica más utilizado en este tipo de estudios. Para captar el movimiento síncrono de las fluctuaciones cíclicas del producto entre los distintos países se utiliza un modelo de factores comunes sintéticos (inobservables), basado en el porcentaje de la varianza del producto que puede atribuirse a dichos factores. Eso nos ayudará a entender las relaciones existentes en la economía mundial.

El esquema del artículo es el siguiente: en un primer epígrafe se describen aspectos referentes a la base de datos utilizada. A continuación, se presentan las técnicas utilizadas para la extracción del ciclo económico y las técnicas factoriales para poder explicar y agrupar los ciclos económicos de los distintos países en ciclos globales comunes. En un tercer apartado se presentan los resultados y finalmente las conclusiones.

\section{BASE DE DATOS}

En esta investigación los datos se han obtenido de The Conference Board and Groningen Growth and Development Centre (GGDC), Total Economy Database, January 2008, http://www.conference-board.org/economics, que ofrece una muestra de países muy completa, organizados por regiones mundiales (Europa occidental, Europa del este, Norte América, Oceanía, América Latina, Oriente Medio, Asia y África), con periodicidad anual desde 1950 hasta 2006, un período lo suficientemente amplio para poder evaluar los ciclos económicos. La gran diversidad de países en cuanto a sus características de tamaño, forma, nivel de desarrollo, 
cultura, geografía, clima, recursos naturales, etc., ha hecho necesario centrar la investigación en el grupo de países que presenten características similares y de mayor interés en el análisis del ciclo económico. Para ello se ha utilizado el criterio del Índice de Desarrollo Humano (IDH) de la Organización de las Naciones Unidas (ONU, 2007) que agrupa a los países en países de desarrollo humano alto, medio y bajo y se ha considerado que es el grupo de países de desarrollo humano alto (países avanzados a partir de ahora) los que constituyen el conjunto de países de mayor interés para este estudio.

En el análisis realizado se han utilizado las series del PIB per cápita real que se presentan a precios de mercado en base 1990 en paridades del poder adquisitivo (PPA) de dólares USA. Como caso especial, para Alemania se ha obtenido el PIB per cápita como la suma de Alemania occidental y de Alemania del este; sin embargo, para el período 1950-1959 los datos inexistentes del PIB en Alemania del este se han interpolado utilizando como tasa de crecimiento la participación media de Alemania del este sobre el total de Alemania, con los datos disponibles.

Las series originales son inicialmente transformadas en logaritmos para eliminar el efecto de las unidades y de posibles outliers, pero no se han tipificado, ya que en este caso la variabilidad de los ciclos es un aspecto importante a tener en cuenta. El siguiente paso consiste en llevar a cabo la extracción del componente cíclico, cuya metodología se presenta en el próximo epígrafe.

\section{Metodología}

Las fuertes tendencias de crecimiento que las economías han presentado en las últimas décadas, han hecho que los trabajos empíricos se centren en el estudio de las expansiones y contracciones de la economía y no en sus «booms» y depresiones, surgiendo el concepto de ciclo económico como las desviaciones que se producen respecto a una tendencia a largo plazo. Por ello, en este artículo se ha optado por la obtención del componente cíclico a partir de los procedimientos de filtrado de Hodrick-Prescott, 1980; Baxter-King, 1995 y filtros de Butterworth. Una vez obtenidos los ciclos de cada país, se utilizarán las técnicas del análisis factorial que tienen como objeto reducir o simplificar un conjunto amplio de datos interdependientes y explicarlos en función de un número pequeño de factores comunes, inobservados e independientes, con la menor pérdida de información posible. Por último, se procederá a realizar el fechado cíclico de los factores comunes, que permitirá realizar un estudio de las características de sus correspondientes fases cíclicas, lo que ofrecerá un entendimiento de los determinantes 
que generan el cambio. A continuación se presentarán los aspectos más destacados de la metodología seguida en este proceso $^{1}$.

\subsection{Descripción de las técnicas de filtrado para la obtención del ciclo en desviaciones respecto a la tendencia.}

Una cuestión latente, que ha suscitado gran controversia, y que se mantiene desde el trabajo de Burns y Mitchell (1946), ha sido la obtención del componente cíclico mediante procedimientos de filtrado. Los procedimientos de filtrado poseen elementos arbitrarios, que no siempre ofrecen resultados adecuados, y utilizar un determinado proceso condiciona claramente los resultados obtenidos. Para la evaluación del ciclo en desviaciones, se aplican los filtros de Hodrick-Prescott (1980), el de Baxter-King (1995) o los filtros de Butterworth derivados de la ingeniería, que a pesar de ir mejorando técnicamente, no aseguran que se extraiga un ciclo incuestionable.

En este trabajo para la presentación de la serie se utiliza el dominio de la frecuencia, una herramienta muy útil para la descomposición de la serie $y_{t}$ es el períodograma o espectro empírico de la serie, que se define como:

$$
h_{y}(w)=|y(w)|^{2} \cdot h_{u}(w)=y(w) \cdot \overline{y(w)} \cdot h_{u}(w)=\psi(L) \psi(F) \sigma_{a}^{2}=\psi\left(e^{-i w}\right) \psi\left(e^{i w}\right) \sigma_{a}^{2},
$$

que permite estudiar la contribución de la varianza o potencia de la serie sobre los distintos armónicos de la frecuencia fundamental o componentes periódicos de una frecuencia determinada. Esto indica la atenuación o amplificación que el filtro introduce para cada frecuencia. De tal forma, si en la representación gráfica el períodograma presenta un «pico» en una determinada frecuencia, indica que dicha frecuencia tiene mayor importancia en la serie que el resto. Formalmente, utilizando la Transformada Discreta de Fourier, se puede descomponer el espectro de la serie $y_{t}$ (Burman, 1980):

$$
h_{y}(w)=h_{T}(w)+h_{C}(w)+h_{E}(w)+h_{u}(w)
$$

lo que equivale a la descomposición de la varianza en el dominio temporal, $\sigma_{y}^{2}=\sigma_{C T}^{2}+\sigma_{E}^{2}+\sigma_{u}^{2}$. Con esta descomposición se puede ver la contribución que tiene

${ }^{1}$ Para unos desarrollos más extensos de la metodología utilizada puede consultarse De Lucas, 2008. 
cada componente en la serie, al asociarles las frecuencias correspondientes como en la figura 1:

- T1 la tendencia asociada a las bajas frecuencias, cuya frecuencia expresada en radianes se encuentra comprendida entre 0 y $2 \pi / 8 s$ (período superior a los ocho años ${ }^{2}$ ), siendo $s$ la frecuencia muestral ( $s=1$ para series anuales). El límite inferior $w=0$ se corresponde con oscilaciones de período infinito, o de duraciones superiores al año, por lo que se le denomina tendencia pura o absoluta.

- El componente cíclico $C_{t}$ está asociado a oscilaciones cuyas frecuencias se encuentran entre los dos y los ocho años, que expresado en radianes sería $[2 \pi / 8 s ; 2 \pi / 2 s]$.

- El componente estacional $E_{t}$ está asociado a un movimiento cuasiperiódico de duración igual al año, que se corresponde con la frecuencia expresada en radianes $2 \pi / s$ y sus correspondientes armónicos $2 \pi m / s$, donde $m=1,2, \ldots, s / 2$.

- El resto de las frecuencias estrictamente inferiores a $2 \pi m / s$ se corresponden con las oscilaciones irregulares, que no deben confundirse con los atípicos o outliers.

Figura 1. Espectro o función de densidad espectral de una serie temporal

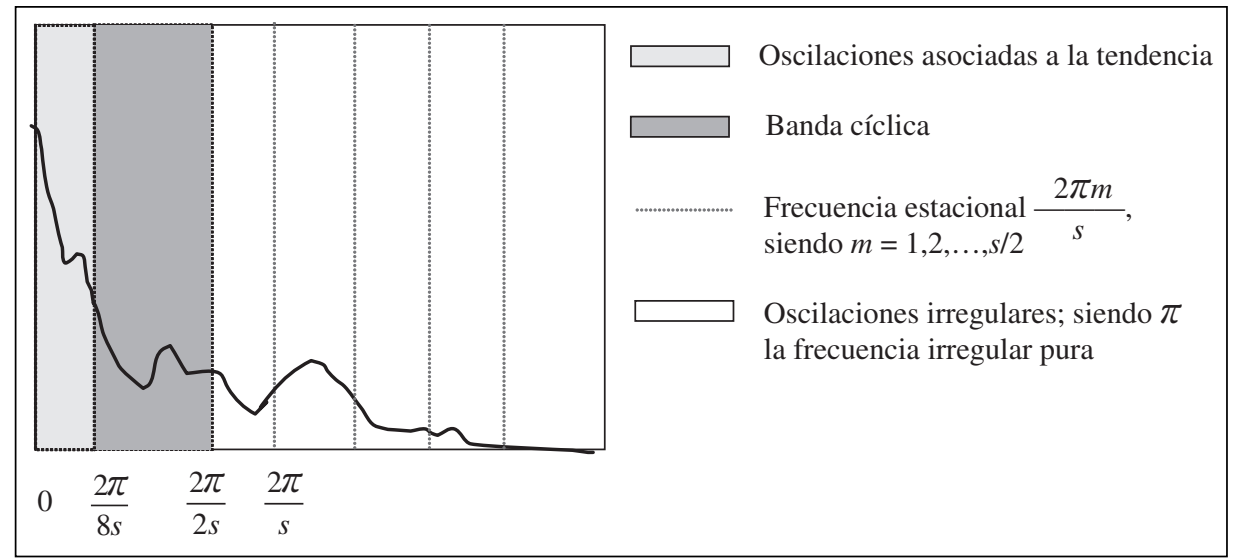

2 Aunque dicho rango puede variar, ya que algunos autores asocian la tendencia con las oscilaciones superiores a los cinco años. 
El enfoque frecuencial considera los filtros como selectores de frecuencias, que permiten extraer parte de la información transportada por la señal. En este caso el proceso de filtrado se define como:

$$
x(w)=h(w) y(w)
$$

donde $h(w)$ es la función de respuesta frecuencial. Si se tratase de un filtro ideal ${ }^{3}$ el funcionamiento sería el siguiente: presenta unas bandas de paso, por las que pasa (no altera) la información de la serie relativa a esas frecuencias. Fuera de esa banda se rechaza (se elimina) la información definida para las frecuencias restantes. Formalmente $h(w)=1$ para las frecuencias seleccionadas y $h(w)=0$ para el resto.

Sin embargo, el problema que presentan los filtros ideales es que aplicados a series en el dominio temporal se definen como filtros de medias móviles infinitos:

$$
H(L)=\frac{w_{p 2}-w_{p 1}}{\pi}+\sum_{k=-\infty}^{\infty} \frac{\operatorname{sen}\left(w_{j} k\right)}{\pi k} L^{h}=a_{0}+\sum_{h=-\infty}^{\infty} a_{k} L^{k},
$$

donde $\mathrm{j}=0, \ldots, \pi$. Pero las series económicas no son infinitas y es necesario truncar el filtro, lo que produce el efecto «leakage» (el filtro no es cero en la frecuencia cero) y el «fenómeno Gibbs» (ripples o rizos en las bandas de frecuencia y rechazo) que introducen comportamientos espúreos.

Todos los filtros de la literatura se pueden analizar como aproximaciones a los filtros ideales, comparando su función de ganancia con la del filtro ideal. En este trabajo se han utilizado los filtros más representativos de la literatura utilizados para la extracción del ciclo económico como un ciclo en desviaciones respecto a la tendencia: I) el filtro de Hodrick y Prescott (1980), en su versión de filtro de paso alto (filtro elimina-

${ }^{3}$ Las propiedades que un filtro ideal debe cumplir son: a) que sea operativo. b) que extraiga el rango específico de periodicidades que se desea, a la vez que deje inalteradas las propiedades del componente extraído. Para el caso que nos ocupa, aísla la frecuencia del ciclo sin introducir cambios en las frecuencias deseadas. c) que tenga ausencia de desplazamiento de la fase (simétricos). Esto implica que los filtros quedarán completamente caracterizados por su función de ganancia en el dominio frecuencial. d) que produzca series estacionarias incluso cuando se aplique a datos con tendencia (series integradas). Equivalentemente, ello implica que la función de respuesta del filtro es cero a la frecuencia cero. e) que no dependa del tamaño muestral, o lo que es lo mismo, que sea invariante en el tiempo. 
dor de la tendencia de las series desestacionalizadas), que se trata de un filtro recursivo básico; II) el de Baxter y King (1995), en su versión de paso banda (pasando por las bandas cíclicas que se definan), que es un filtro de medias móviles simétrico; y III) los filtros de la familia Butterworth, también en su versión de paso banda, que son filtros recursivos con estructuras ARMA. Estos últimos filtros engloban como caso particular al filtro de Hodrick-Prescott (1980), para la extracción de la tendencia; y además son más eficientes que el filtro MA de Baxter-King (1995), puesto que no generan ciclos espurios debidos a los rizos en las bandas de paso y rechazo. Una comparación de los filtros a través de su función de ganancia se puede observar en la figura 2.

Figura 2. Comparación de las funciones de ganancia de los distintos filtros

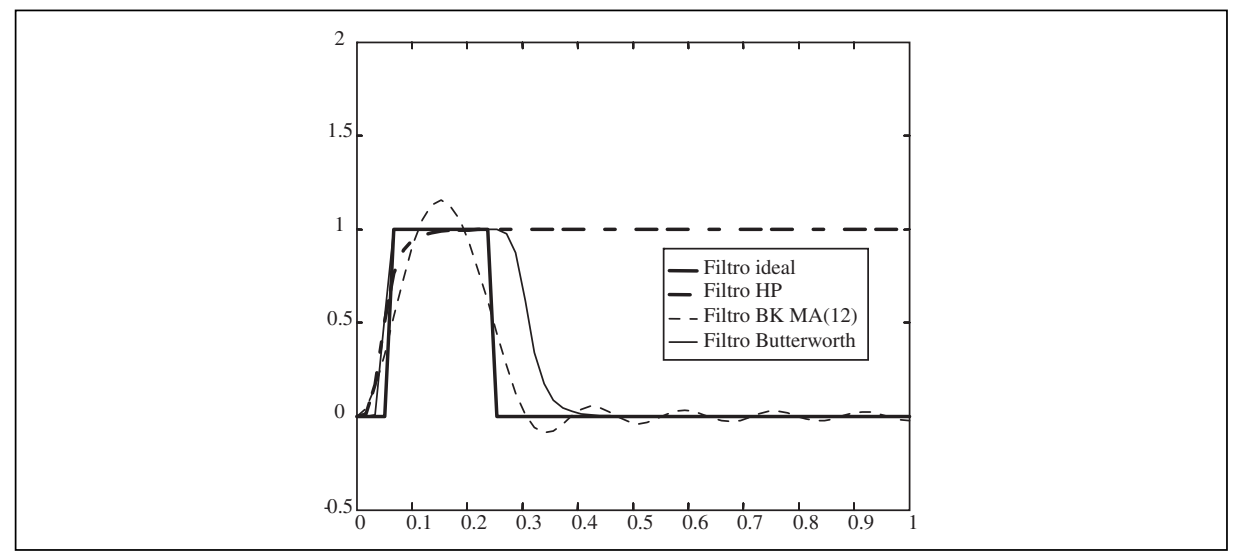

Las conclusiones generales que se pueden extraer sobre los filtros señalados, son que cumplen la mayoría de las propiedades de un filtro deseable. Sin embargo, dichos filtros son óptimos cuando al filtrar por la serie $y_{t}$ lo restante es ruido blanco, y esto no se cumple en la mayoría de las series económicas que presentan tendencia (Estrella, 2007) como se muestra en la figura 3. Esto podría tener consecuencias importantes sobre el filtrado ya que incluso el filtro ideal adopta una estructura que puede inducir ciclos espurios al no extraer adecuadamente la banda cíclica, atenuando frecuencias cíclicas a la vez que acentúa la banda cíclica por encima del valor uno en las frecuencias próximas a los ocho años.

En definitiva, las conclusiones extraídas no son determinantes en cuanto a qué filtro utilizar en la práctica, por ello, se ha tomado la decisión de realizar la estimación de los ciclos con los tres filtros descritos y emplearlos en la estimación de los ciclos globales mediante 
análisis factorial como se describe en el siguiente epígrafe. De este modo se ofrece una visión más amplia sobre el comportamiento de las series y permite comparar los resultados.

Figura 3. Funciones de ganancia de los filtros afectadas por distintas estructuras

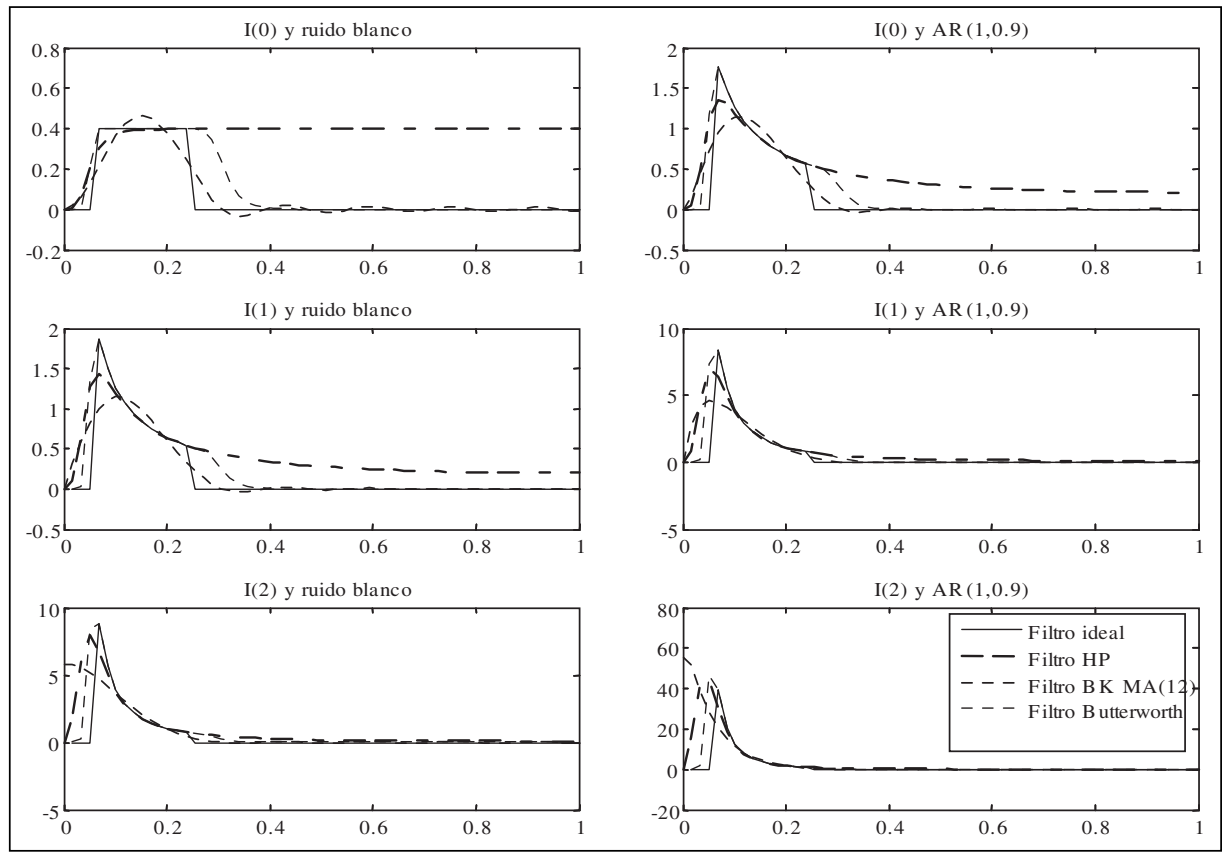

\subsection{Estimación del ciclo internacional mediante el análisis factorial}

La referencia fundamental para el análisis factorial de los ciclos económicos son los trabajos de Stock y Watson $(1999,2002)$. El método estadístico se basa en asumir a priori una estructura finita de retardos en las representaciones de las variables bajo estudio en función de los factores no observables, lo que permite utilizar un análisis de componentes principales en el cálculo de los factores. El uso de componentes principales facilita el cálculo de los factores cuando el número de variables explicativas es grande y permite lidiar con irregularidades en los datos.

Formalmente, en el modelo más general que presentan, se asume que un conjunto de variables $X_{t}$ puede ser representada por factores comunes subyacentes, como: 


$$
\mathrm{XT}=\Lambda_{t} F_{t}+e_{t}
$$

donde $\operatorname{los} N \times 1$ componentes específicos, $e_{t}=\left(e_{1, t}, e_{2, t}, \ldots, e_{N, t}\right)^{\prime}$, están en general serialmente correlados y con otras variables del modelo. Además, las cargas factoriales $\Lambda_{t}(N \times r)$ varían en el tiempo de acuerdo a una estructura de medias móviles:

$$
\Lambda_{t}=\Lambda_{t-1}+h \xi_{t}
$$

siendo $h$ una matriz diagonal $N \times N$, y $\xi_{t} \operatorname{los} r \times 1$ errores estocásticos.

Sin embargo, para la estimación del modelo 5-6 con un número muy elevado de series (cuando $N$ es grande), que es el caso que tratamos en este trabajo, Stock y Watson (1989, 2002) proponen un enfoque de estimación que denominan quasi-verosímil, ya que hay que realizar supuestos muy restrictivos sobre el modelo, tal que $\Lambda_{t}=\Lambda, h=$ 0 y $e_{i, t}$ son i.i.d. como $N\left(0, \sigma_{e}^{2}\right)$. Con estos supuestos se pueden obtener estimaciones consistentes de los factores y las cargas, $(\Lambda, F)$, mediante la resolución de un problema de mínimos cuadrados no lineales:

$$
\mathrm{V}(\tilde{F}, \tilde{\Lambda})=(N T)^{-1} \sum_{i=1}^{N} \sum_{t=1}^{T}\left(x_{i t}-\tilde{\lambda}_{i} \tilde{F}_{t}\right) 2
$$

tal que (7) es la función objetivo de los supuestos valores de los factores, $\tilde{F}_{t}=\left(\tilde{F}_{1, t}, \ldots\right.$, $\left.\tilde{F}_{T}\right)^{\prime}$ y de las cargas $\tilde{\Lambda}$. Donde $\hat{F}$ y $\hat{\Lambda}$ son los valores resultantes de la minimización eficiente de (7). Además, como $N<T$, el problema de minimización es equivalente a la maximización de $\operatorname{tr}\left[\Lambda X^{\prime} X \Lambda\right]$ sujeto a $\Lambda^{\prime} \Lambda / N=I_{r}$; cuya solución lleva al clásico análisis de componentes principales, cuya descripción detallada se puede revisar en Peña (2002). Los factores se seleccionan utilizando los correspondientes autovectores de los $k$ autovalores más grandes de la matriz $T \times T$ :

$$
\frac{1}{N} \sum_{i=1}^{N} x_{i} x_{i}^{\prime}
$$

siendo la matriz de covarianzas de $X$. Esta propuesta de computación sería equivalente a la de un modelo factorial estático, ya que sólo se utiliza la relación contemporánea entre los países, por lo que estaríamos hablando del llamado índice de referencia de Stock y Watson (1989). 
En definitiva, en este trabajo la propuesta metodológica es que el análisis factorial se realizará en dos etapas: 1) se estimará el modelo con todos los países de la submuestra correspondiente y seleccionando el número de factores de acuerdo a los resultados del modelo en función del gráfico de sedimentación y del porcentaje total de varianza del modelo. 2) Se irán eliminando aquellos países que no tengan una carga factorial con un peso aceptable $\left(l_{j i}\right.$ $\geq 0.4$ ó $l_{j i} \geq 0.3$ ) en ninguno de los factores, procediendo a las reestimaciones del modelo. Este procedimiento se repetirá hasta que se obtengan factores más o menos interpretables, con cargas factoriales y comunalidades con un peso razonable $\mathrm{y}$, cuando los contrastes tradicionalmente utilizados en la literatura muestren la adecuación de los datos al modelo: (a) que el determinante de la matriz de covarianzas sea suficientemente pequeño, b) el contraste de esferidad de Barlett que plantea la hipótesis nula de que las variables originales no están correlacionadas, y c) el índice de Kaiser-Meyer-Olkin (KMO) ${ }^{4}$.

\subsection{Fechado de los ciclos comunes}

A los puntos máximos y mínimos del ciclo se les denomina puntos de giro o inflexión, ya que marcan el cambio de una fase de expansión a una de desaceleración, o de una fase de recesión a una de recuperación. La identificación o fechado de estos puntos determina la cronología básica del ciclo, y es de gran interés para los especialistas, ya que determina el «ritmo» de las fluctuaciones que registra la serie objeto de estudio. Consecuentemente, la cronología o fechado cíclico permite además realizar un estudio de las características de las fases cíclicas, que se puede resumir en cuatro medidas fundamentales ${ }^{5}$ :

- Duración: la duración de una expansión corresponde al tiempo que transcurre desde el valle, que es el nivel más bajo de actividad alcanzado al final de la recesión, hasta el siguiente pico, que es el punto más alto alcanzado por expansión de la actividad económica. Por lo tanto, la suma de la duración de una expansión $\left(d^{+}\right)$y de una recesión $\left(d^{-}\right)$correspondería a la duración del ciclo.

- Amplitud: se corresponde a la diferencia, en valor absoluto, entre el valor de la señal cíclica en el mínimo y en el máximo anterior. En el caso de una expansión, la amplitud corresponde a la ganancia en términos de producción durante un período de expansión; mientras, en el caso de recesión, se puede interpretar como el porcentaje de pérdida.

\footnotetext{
${ }^{4}$ Para ver en detalle estos contrastes consultar Peña (2002).

5 Para analizar otras medidas véase Harding y Pagan (2002), Camacho, Pérez-Quiros y Saiz (2005).
} 
- Intensidad: es el cociente de la amplitud sobre la duración de una fase expansiva o recesiva.

- Asimetría: se refiere a si el componente tendencial se comporta de igual manera cuando está por encima o por debajo de su tendencia. Por lo tanto, el ciclo será simétrico cuando $d^{-}=d^{+}$.

En la práctica el fechado cíclico de manera manual es una tarea tediosa y susceptible de error, por lo que se han desarrollado algunos programas ${ }^{6}$. En este trabajo se utiliza el método de Harding y Pagan (2002), implementado en el programa de libre acceso Scilab ${ }^{7}$. Este método define los puntos de giro como aquellos valores donde la tangente es nula, con lo que se están identificando las fases de expansión o desaceleración de la economía cuando no cambian de signo; mientras que identifica las expansiones y recesiones cuando se cruza la línea del cero. Para ello se llevan a cabo las siguientes fases: 1) determinación de los potenciales puntos de giro de una serie, definiendo un máximo local como aquel que ocurre al tiempo $t$ si $\left\{y_{t}\right\rangle$ $\left.y_{t+k}\right\}$ (y viceversa si se trata de un mínimo), 2) fijando $k=3$ en el caso de datos anuales, para asegurar que los picos y valles se alternan y 3 ) se debe cumplir que al menos la duración de una fase debe ser de un año y un ciclo completo como mínimo debe ser de dos años.

\section{Resultados}

En este apartado se presentan los resultados de los ciclos comunes obtenidos del análisis factorial realizado con los ciclos específicos del PIB per cápita de los países avanzados ofrecidos en la base GDDC (2007), con un período muestral anual de 1950 a 2006. A partir de los resultados obtenidos tras la aplicación de los tres tipos de filtro analizados, se procederá a evaluar los resultados del modelo factorial, y a continuación, se analiza el fechado de los ciclos comunes obtenidos de dicho análisis.

En una primera etapa, se procedió a realizar las estimaciones preliminares con todos los países comprobando que han dado lugar a un número similar de factores ${ }^{8}$. Para

${ }^{6}$ El programa más extendido el de Bry-Boschan (1971), que utiliza el NBER. Este programa fecha los puntos de giro como los cambios en la derivada respecto a $t$, lo que en un ciclo en desviaciones se corresponderían con los puntos de giro de los ciclos clásicos.

7 Se puede descargar en la dirección http://www.scilab.org/

${ }^{8}$ Los resultados obtenidos en las estimaciones preliminares y la muestra completa de países avanzados utilizada pueden consultarse en De Lucas, 2008. 
los tres ciclos analizados (HP, BK y BUTT) se obtiene un primer ciclo común más representativo que recoge un número importante de países con cargas factoriales con un peso razonable en el factor. Las diferencias en este primer factor entre los tres ciclos son debidas a que no incluye a los países de la Europa del este que vendrían recogidos en el segundo factor, junto con algún país de Oriente Próximo y de Latinoamérica con un comportamiento opuesto a los primeros. El resto de los factores no tienen una explicación razonable en ninguno de los tres modelos y pueden haberse formado debido a la gran disparidad existente entre los países no clasificados en los dos primeros factores, por lo que no se deberían considerar en el modelo de factores comunes. Por otro lado, tanto el análisis de las cargas factoriales y las comunalidades, como los criterios de varianza y los tests de adecuación de la muestra reflejan que es necesario eliminar del modelo aquellos países que no forman parte de o no aportan información sobre los posibles ciclos comunes globales. Una vez eliminados aquellos países que no son relevantes en el modelo factorial se obtienen los resultados definitivos, cuya adecuación es confirmada por los diferentes tests, que se presentan en las tablas de resultados 1-3.

Los resultados definitivos de HP se presentan en la tabla 1. Los resultados muestran un primer ciclo común que incluye la mayor parte de los países de la OCDE sin Noruega, Japón, Qatar, Arabia Saudita, Nueva Zelanda y Corea del sur, y de la UE sin Chipre y Malta, junto con el resto de los países de Europa del este, Barbados (paraíso fiscal con gran dependencia económica de Estados Unidos y Europa) y Arabia Saudita (líder de la OPEP). En el análisis realizado es Francia el país de referencia (país con mayor carga factorial en el primer factor). El segundo factor es complicado de explicar, pero se podría interpretar como un ciclo específico que recoge el comportamiento de países con un crecimiento superior (cuando el signo de la carga es positivo) a los del primer factor que ante la crisis de 1991 aumentaron su crecimiento, frente a la disminución que experimentaron la mayoría de los países industrializados, entre los que se encuentran Malasia que se ve más influenciados por los países de la UE, representados por Luxemburgo, mientras que Cuba se ve más desbordada por el ciclo de los países de la Europa del este, que presentan un crecimiento inferior a los del primer factor (al ser el signo de sus cargas negativo). Por la dificultad de interpretar el segundo factor, se valoró la posibilidad de eliminar los países de este grupo de la estimación y sólo considerar un único factor, pero la estimación sin estos países no explica un porcentaje de varianza total suficiente para que el modelo sea adecuado, por lo que se ha mantenido la estimación con los dos factores señalados. 
Tabla 1. Modelo factorial final con dos factores de los ciclos de Hodrick-Prescott

\begin{tabular}{|c|c|c|c|}
\hline \multirow[b]{2}{*}{ Países } & \multicolumn{2}{|c|}{ cargas } & \multirow[b]{2}{*}{ Comunalidades } \\
\hline & factor 1 & factor 2 & \\
\hline Austria & 0.3842 & 0.4706 & 0.3691 \\
\hline Bélgica & 0.6080 & 0.6420 & 0.7818 \\
\hline Dinam arca & 0.4090 & -0.2770 & 0.2441 \\
\hline Finlandia & 0.8075 & -0.0005 & 0.6521 \\
\hline Francia & 0.7025 & 0.5486 & 0.7946 \\
\hline Alemania & 0.4219 & 0.3836 & 0.3251 \\
\hline Grecia & 0.5532 & 0.3105 & 0.4025 \\
\hline Islandia & 0.4558 & -0.0196 & 0.2082 \\
\hline Irlanda & 0.4574 & 0.4195 & 0.3852 \\
\hline Italia & 0.4082 & 0.3911 & 0.3196 \\
\hline Luxemburgo & 0.5030 & 0.4414 & 0.4479 \\
\hline Países Bajos & 0.5548 & 0.4794 & 0.5376 \\
\hline Portugal & 0.5288 & 0.6741 & 0.7340 \\
\hline España & 0.4397 & 0.3464 & 0.3134 \\
\hline Suecia & 0.8118 & -0.1202 & 0.6734 \\
\hline Suiza & 0.6350 & 0.4756 & 0.6294 \\
\hline Reino Unido & 0.8262 & -0.1148 & 0.6958 \\
\hline Canadá & 0.7453 & -0.1151 & 0.5688 \\
\hline Estados Unidos & 0.5811 & -0.0243 & 0.3383 \\
\hline Australia & 0.5929 & -0.0166 & 0.3518 \\
\hline Albania & 0.6206 & -0.5036 & 0.6388 \\
\hline Bulgaria & 0.2870 & -0.5979 & 0.4398 \\
\hline Checoslovaquia & 0.4971 & -0.5478 & 0.5472 \\
\hline Hungría & 0.6279 & -0.5537 & 0.7009 \\
\hline Polonia & 0.5138 & -0.4958 & 0.5098 \\
\hline Rumanía & 0.3938 & -0.6002 & 0.5153 \\
\hline Yugoslavia & 0.7034 & -0.3489 & 0.6164 \\
\hline URS S & 0.5239 & -0.0743 & 0.2800 \\
\hline Japón & 0.2446 & 0.4695 & 0.2803 \\
\hline Malasia & -0.3582 & 0.4195 & 0.3043 \\
\hline Singapur & 0.0747 & 0.4907 & 0.2463 \\
\hline Barbados & 0.8146 & -0.0975 & 0.6731 \\
\hline Chile & -0.0342 & 0.4592 & 0.2121 \\
\hline Cuba & 0.5072 & -0.2936 & 0.3434 \\
\hline México & -0.1151 & 0.5369 & 0.3015 \\
\hline Qatar & 0.0568 & 0.4903 & 0.2437 \\
\hline Arabia Saudita & -0.2821 & 0.7183 & 0.5955 \\
\hline Emiratos Árabes & -0.2867 & 0.5464 & 0.3808 \\
\hline \multicolumn{3}{|c|}{ Adecuación muestral de Kaiser-Meyer-Olkin: } & 0.608 \\
\hline \multicolumn{3}{|c|}{ Determinante de la matriz de correlaciones } & $3,17 \mathrm{E}-023$ \\
\hline \multicolumn{4}{|c|}{ Prueba de esfericidad de Bartlett } \\
\hline & \multicolumn{2}{|c|}{ Chi-cuadrado } & 2201.691118 \\
\hline & & gl & 703 \\
\hline & & Sig. & 0.00 \\
\hline
\end{tabular}

Nota: en negro aparecen las cargas con una suficiente correlación con el factor o factores. Lo mismo ocurre para las comunalidades según presenten suficiente porcentaje de varianza explicado por el factor para ese país o no. 
Los resultados del modelo de BK, tabla 2, muestran un primer factor común a los países de la OCDE, sin España, Islandia, Nueva Zelanda, Hungría, Polonia, República Checa y Corea del sur, y de la UE sin Chipre, España y Malta; junto con Yugoslavia, URSS, Hong-Kong, Malasia, Singapur, Barbados, Brasil, Israel y Emiratos Árabes; siendo Suiza el país de referencia en dicho ciclo. El segundo factor, mucho más claro en este modelo, representa el ciclo de los países de la Europa del este sin Yugoslavia y URSS, junto con países con un comportamiento en contra de este ciclo, como México, Trinidad y Tobago, Arabia Saudí y Emiratos Árabes.

Finalmente, los resultados para BUTT, recogidos en la tabla 3, son muy similares a los obtenidos con BK, pero más robustos y adecuados si se comparan los test de ajuste, y con menor pérdida de información al incluir más países en el modelo. El primer factor recoge de nuevo un ciclo para los países de la OCDE, sin Islandia, Noruega y Nueva Zelanda, y de la UE sin Chipre; junto con Yugoslavia, Hong-Kong, Malasia, Singapur, Barbados, Brasil, Chile e Israel; siendo de nuevo Bélgica la serie de referencia del ciclo común. El segundo factor también representa el ciclo de los países de la Europa del este sin Yugoslavia y URSS (que ha sido eliminada del modelo), junto con países con un comportamiento en contra de este ciclo, como México, Trinidad y Tobago, Arabia Saudí y Emiratos Árabes.

Respecto a la interpretación de los ciclos, es claro que gran parte de los países de la OCDE y de la UE, junto con alguno de los países más desarrollados de Latinoamérica, Asia y Oriente Medio, siguen un ciclo global común. Esto confirma la idea, analizada en muchos trabajos, de que un factor común internacional es el determinante de los ciclos económicos de los países industrializados. Los factores de HP, BK y BUTT presentan también un primer ciclo global común de los países más avanzados; mientras que el segundo representa un ciclo de países algo retardados respecto a los que componen el primer factor, con fluctuaciones más suaves y menos afectados por las crisis y/o recesiones mundiales, salvo para el caso de la crisis de 1991, que aunque llega más tarde a estos países es mucho más intensa. 
Tabla 2. Modelo factorial final con dos factores de los ciclos de Baxter-King

\begin{tabular}{|c|c|c|c|}
\hline \multirow[b]{2}{*}{ Países } & \multicolumn{2}{|c|}{ cargas } & \multirow[b]{2}{*}{ Comunalidades } \\
\hline & factor 1 & factor 2 & \\
\hline Austria & 0.6176 & 0.1367 & 0.4001 \\
\hline Bélgica & 0.8537 & 0.2322 & 0.7828 \\
\hline Dinamarca & 0.4064 & -0.0554 & 0.1682 \\
\hline Finlandia & 0.6854 & -0.2711 & 0.5433 \\
\hline Francia & 0.7640 & 0.0994 & 0.5936 \\
\hline Alemania & 0.7092 & 0.0759 & 0.5087 \\
\hline Grecia & 0.3110 & -0.0369 & 0.0981 \\
\hline Irlanda & 0.4612 & 0.0818 & 0.2194 \\
\hline Italia & 0.5079 & 0.1595 & 0.2834 \\
\hline Luxemburgo & 0.6487 & 0.1463 & 0.4422 \\
\hline Países Bajos & 0.6898 & 0.1578 & 0.5007 \\
\hline Noruega & 0.3126 & 0.0995 & 0.1076 \\
\hline Portugal & 0.5633 & 0.3147 & 0.4163 \\
\hline Suecia & 0.5997 & -0.1683 & 0.3880 \\
\hline Suiza & 0.7715 & 0.2096 & 0.6391 \\
\hline Reino Unido & 0.6585 & -0.3287 & 0.5417 \\
\hline Canadá & 0.4959 & -0.1632 & 0.2726 \\
\hline Estados Unidos & 0.5309 & -0.1030 & 0.2925 \\
\hline Australia & 0.3212 & -0.1977 & 0.1422 \\
\hline Albania & 0.2496 & -0.6875 & 0.5349 \\
\hline Bulgaria & -0.0039 & -0.6794 & 0.4616 \\
\hline Checoslovaquia & 0.3389 & -0.6538 & 0.5424 \\
\hline Hungría & 0.1697 & -0.6625 & 0.4677 \\
\hline Polonia & 0.2117 & -0.6339 & 0.4467 \\
\hline Rumanía & 0.1227 & -0.7246 & 0.5400 \\
\hline Yugoslavia & 0.3480 & -0.2938 & 0.2075 \\
\hline URSS & 0.3815 & 0.0563 & 0.1487 \\
\hline Hong Kong & 0.3885 & 0.1849 & 0.1851 \\
\hline Japón & 0.5490 & 0.2009 & 0.3418 \\
\hline Malasia & 0.4988 & 0.2913 & 0.3337 \\
\hline Singapur & 0.3870 & -0.0097 & 0.1499 \\
\hline Barbados & 0.5447 & -0.4106 & 0.4653 \\
\hline Brasil & 0.3842 & -0.2695 & 0.2203 \\
\hline México & 0.3176 & 0.3495 & 0.2231 \\
\hline Trinidad \& Tobago & 0.2878 & 0.3399 & 0.1984 \\
\hline Israel & 0.3255 & 0.0074 & 0.1060 \\
\hline Arabia Saudita & 0.1860 & 0.6293 & 0.4306 \\
\hline Emiratos Árabes & 0.3620 & 0.3131 & 0.2291 \\
\hline \multicolumn{4}{|c|}{ VARIANZA EXPLICADA POR EL MODELO: $35.72 \%$} \\
\hline \multicolumn{3}{|c|}{ Adecuación muestral de Kaiser-Meyer-Olkin: } & 0.523 \\
\hline \multicolumn{3}{|c|}{ Determinante de la matriz de correlaciones } & $9.18 \mathrm{E}-17$ \\
\hline \multicolumn{4}{|c|}{ Prueba de esfericidad de Bartlett } \\
\hline & \multicolumn{2}{|c|}{ Chi-cuadrado } & 1569.378596 \\
\hline & & gl & 703 \\
\hline & & Sig. & 0.00 \\
\hline
\end{tabular}

Nota: en negro aparecen las cargas con una suficiente correlación con el factor o factores. Lo mismo ocurre para las comunalidades según presenten suficiente porcentaje de varianza explicado por el factor para ese país o no. 
Tabla 3. Modelo factorial final con dos factores de los ciclos de Butterworth

\begin{tabular}{|c|c|c|c|}
\hline \multirow[b]{2}{*}{ Países } & \multicolumn{2}{|c|}{ cargas } & \multirow[b]{2}{*}{ Comunalidades } \\
\hline & factor 1 & factor 2 & \\
\hline Austria & 0.5935 & 0.3408 & 0.4684 \\
\hline Bélgica & 0.8375 & 0.2664 & 0.7723 \\
\hline Dinamarca & 0.3217 & -0.1549 & 0.1275 \\
\hline Finlandia & 0.7469 & -0.1552 & 0.5819 \\
\hline Francia & 0.7699 & 0.2085 & 0.6363 \\
\hline Alemania & 0.6025 & 0.2047 & 0.4049 \\
\hline Grecia & 0.3851 & 0.0555 & 0.1514 \\
\hline Irlanda & 0.5360 & 0.1849 & 0.3215 \\
\hline Italia & 0.5296 & 0.0859 & 0.2879 \\
\hline Luxemburgo & 0.5191 & 0.2573 & 0.3357 \\
\hline Países Bajos & 0.6031 & 0.2355 & 0.4193 \\
\hline Portugal & 0.6484 & 0.2934 & 0.5065 \\
\hline España & 0.3263 & 0.2021 & 0.1473 \\
\hline Suecia & 0.6520 & -0.0807 & 0.4316 \\
\hline Suiza & 0.7920 & 0.3054 & 0.7205 \\
\hline Reino Unido & 0.6622 & -0.4024 & 0.6005 \\
\hline Canadá & 0.5194 & -0.2951 & 0.3569 \\
\hline Estados Unidos & 0.5268 & -0.2312 & 0.3310 \\
\hline Australia & 0.4071 & -0.2520 & 0.2292 \\
\hline Albania & 0.3460 & -0.6806 & 0.5829 \\
\hline Bulgaria & 0.0767 & -0.6275 & 0.3996 \\
\hline Checoslovaquia & 0.3301 & -0.6954 & 0.5925 \\
\hline Hungría & 0.2702 & -0.6905 & 0.5498 \\
\hline Polonia & 0.2017 & -0.7072 & 0.5408 \\
\hline Rumanía & 0.2077 & -0.7738 & 0.6418 \\
\hline Yugoslavia & 0.4644 & -0.2783 & 0.2932 \\
\hline Hong Kong & 0.3526 & 0.0883 & 0.1321 \\
\hline Japón & 0.5185 & 0.1539 & 0.2926 \\
\hline Malasia & 0.3510 & 0.2257 & 0.1741 \\
\hline Singapur & 0.3832 & 0.0542 & 0.1498 \\
\hline Barbados & 0.6276 & -0.4628 & 0.6080 \\
\hline Brasil & 0.3684 & -0.4043 & 0.2992 \\
\hline Chile & 0.3561 & 0.0168 & 0.1271 \\
\hline México & 0.3401 & 0.4799 & 0.3460 \\
\hline Trinidad \& Tobago & 0.3569 & 0.2883 & 0.2105 \\
\hline Israel & 0.3477 & 0.0827 & 0.1277 \\
\hline Arabia Saudita & 0.2369 & 0.5727 & 0.3841 \\
\hline Emiratos Árabes & 0.3894 & 0.3544 & 0.2773 \\
\hline \multicolumn{4}{|c|}{ VARIANZA EXPLICADA POR EL MODELO: $38.31 \%$} \\
\hline \multicolumn{3}{|c|}{ Adecuación muestral de Kaiser-Meyer-Olkin: } & 0.496 \\
\hline \multicolumn{3}{|c|}{ Determinante de la matriz de correlaciones } & $2.45 \mathrm{E}-19$ \\
\hline \multicolumn{4}{|c|}{ Prueba de esfericidad de Bartlett } \\
\hline & \multicolumn{2}{|c|}{ Chi-cuadrado aproximado } & 1821.192448 \\
\hline & & gl & 703 \\
\hline & & Sig. & 0.00 \\
\hline
\end{tabular}

Nota: en negro aparecen las cargas con una suficiente correlación con el factor o factores. Lo mismo ocurre para las comunalidades según presenten suficiente porcentaje de varianza explicado por el factor para ese país o no. 
En resumen, en los tres modelos definitivos no se pueden considerar parte de un comportamiento común a países como Chipre, Malta, Nueva Zelanda, Corea del Sur, Argentina, Costa Rica, Uruguay, Bahréin, Kuwait y Omán. Por otro lado, países como Islandia, Irlanda, Noruega, URSS, Chile, Cuba, Trinidad y Tobago, Qatar y Emiratos Árabes no son países que formen parte de los ciclos globales de los tres modelos, por lo que en estos casos las técnicas de filtrado condicionan los resultados respecto a estos países. Del análisis gráfico de los factores comunes, Anexo 1, se concluye los tres modelos utilizados presentan un primer ciclo global común de los países más avanzados, mientras que el segundo representa un ciclo específico de países algo retardados respecto a los que componen el primer factor, con fluctuaciones más suaves y menos afectados por las crisis y/o recesiones mundiales, salvo para el caso de la crisis de 1991, que aunque llega más tarde a estos países es mucho más intensa.

Respecto al fechado cíclico, en general, los resultados del análisis confirman, desde una perspectiva histórica, la identificación de las dos últimas etapas del siglo XX señaladas en la literatura y que coinciden en general en las fases de expansión y contracción con la cronología del NBER para Estados Unidos ${ }^{9}$; siempre teniendo en cuenta que la cronología del NBER se refiere a los ciclos en niveles y aquí se analizan ciclos en tasas y en desviaciones (que adelantan o retardan a los ciclos en niveles como se ha comentado anteriormente). Respecto a las características de las fases cíclicas, se puede concluir que, en general, existe una mayor duración de las fases expansivas que de las contractivas en los países industrializados; sin embargo estas conclusiones respecto a la asimetría de los ciclos no son tan claras cuando se analizan los países emergentes o en desarrollo.

En cuanto a la sincronización entre los ciclos de cada país y los ciclos comunes estimados, en general, se observa en las figuras del anexo 1, una mayor sincronización en las contracciones mundiales que en las expansiones. Además, cabría esperar una mayor sincronización con el transcurrir del tiempo. No obstante, se puede observar un debilitamiento de la sincronización a nivel global a partir de los años ochenta en los países industrializados, mientras que en los países emergentes y en desarrollo la interrelación con los ciclos globales se ha mantenido más o menos parecida en todo el período analizado.

En general, los resultados del análisis confirman, desde una perspectiva histórica, la identificación de las dos últimas etapas del siglo xx señaladas en la literatura y que

9 La cronología del NBER se puede consultar desde 1850 hasta la actualidad, en datos trimestrales, en http://www.nber.org/cycles.html 
coinciden a grandes rasgos con la cronología del NBER: I) en la etapa 1950-1973, denominada «edad de oro» (Solomou, 1998), se produce una estabilización del crecimiento que se evidencia con ciclos más suaves (menor amplitud) y de menor duración que en la etapa posterior; II) la etapa a partir de los años setenta presentan fluctuaciones más prolongadas (mayor duración) y más pronunciadas (mayor amplitud).

Tabla 4. Propiedades de los ciclos comunes: duración, amplitud y asimetrías

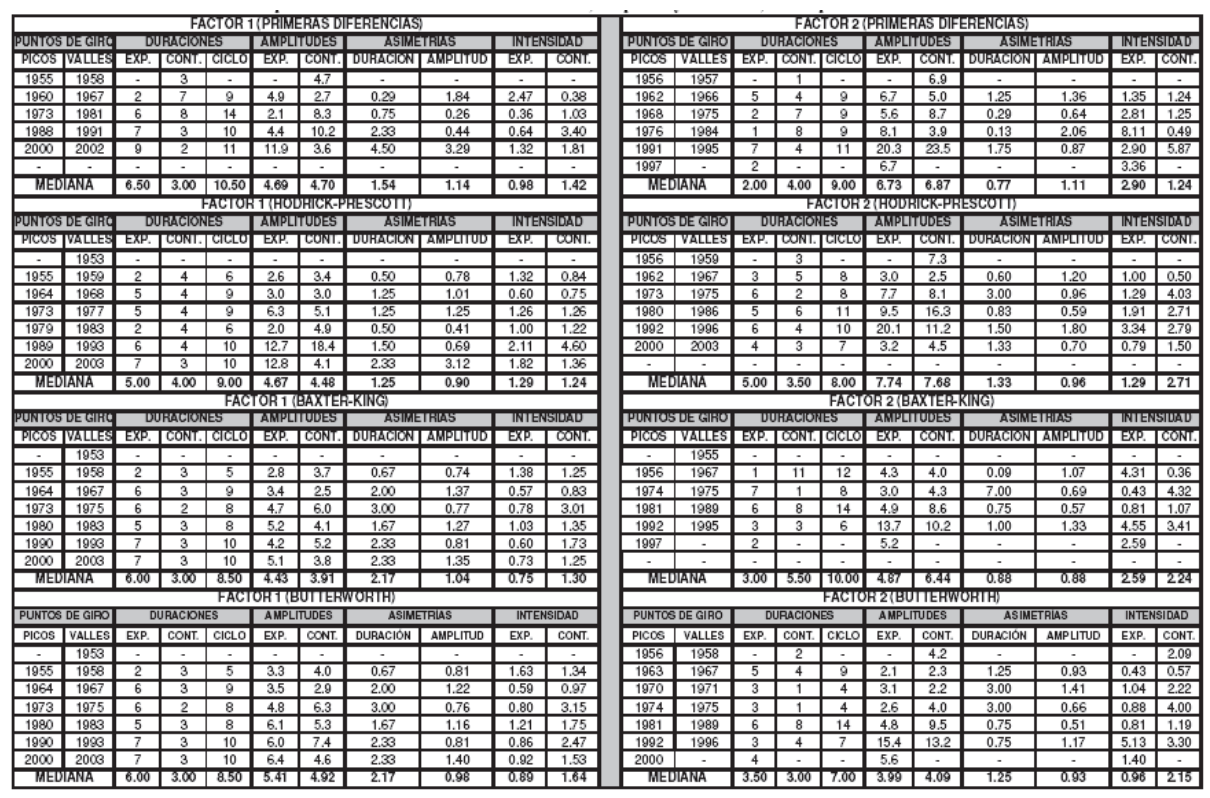

Las fases identificadas en el primer factor común, tabla 4, muestran las dos pequeñas desaceleraciones en el período 1950-1973, que se producen en Estados Unidos en 1954 y 1961; con una clara clasificación de las recesiones de 1974-75, 1981, 1991, 2001; mientras que las desaceleraciones internacionales de 1986 y 1995 sólo son claras en los resultados de BK y HP. El segundo ciclo, representa un comportamiento contrario de Hong-Kong y Malasia frente al del primer ciclo con los países más avanzados, con un sorprendente crecimiento en 1991 (crisis internacional). En cuanto a las características de las fases de los ciclos comunes, se puede señalar que el primer factor en promedio presenta, en los cuatro casos, asimetrías en las duraciones, con expansiones más prolongadas que las contracciones. Por otro lado, el segundo factor es más asimétrico respecto a la amplitud que a las duraciones y las conclusiones sobre el comportamiento asimétrico de las expansiones ya no son tan claras. 


\section{Conclusiones}

En este artículo se ha tratado de describir en qué medida los países se interrelacionan, o bien, presentan comovimientos en un ciclo internacional. Para ello, primero se ha procedido a una revisión de las técnicas de filtrado de los ciclos, que ha permitido comparar las metodologías empleadas y ofrecer un evidencia más amplia sobre los resultados obtenidos. Por ello se ha decidido presentar los diferentes resultados con los tres métodos de filtrado más conocidos en la literatura de los ciclos económicos. Por otro lado, con una gran muestra de países tan diversos, se ha considerado oportuno, tratar de resumir la evolución de los países mediante las técnicas multivariantes del análisis factorial, con la mínima pérdida de información posible.

En este artículo se confirma la hipótesis, analizada en muchos trabajos, de que un factor común internacional es el determinante de los ciclos económicos de los países industrializados. Mediante el análisis factorial se ha encontrado, sin imponer restricciones a priori, un ciclo global de los países más avanzados. Finalmente se detectan los puntos de giro de las dos últimas etapas del siglo $\mathrm{xx}$; una primera etapa que abarca la época de postguerra hasta las crisis del petróleo, que ha reflejado una época de estabilidad económica y de ciclos más suaves y menos duraderos; frente a las fluctuaciones cíclicas más intensas y prolongadas del último tercio del siglo xx y los primeros años del actual, debidas a los vaivenes de la economía con varias recesiones (1974-1975, 1981-82, 1991 y 2001) y contracciones mundiales (1986 y 1995), que han afectado de manera diferente a las distintas regiones mundiales. Además, el análisis de la relación entre los ciclos de cada país y los ciclos comunes estimados han reflejado una mayor sincronización en las contracciones mundiales que en las expansiones en los países industrializados, y aunque se esperaría una mayor sincronización de los ciclos en las últimas décadas, por el contrario, se puede observar un debilitamiento de los comovimientos a nivel internacional en los países industrializados.

\section{REFERENCIAS BIBLIOGRÁFICAS}

Artis, M.; Marcellino, M. y Proietti, T. (2004), «Dating Business Cycles: A Methodological Contribution with an Application to the Euro Area», Oxford Bulletin fo Economics and Statistics, 66, 4, 537-565.

BAXter, M y King, R. (1995), «Measuring Business Cycles Approximate Band-Pass Filter for Economic Time Series», NBER working paper series, $\mathrm{n}^{\circ}$ 5022, Cambridge, Massachusetts. 
Bry, G. y Boschan, C. (1971), «Cyclical Analysis of Time Series: Selected Procedures and Computer Programs», NBER Technical Paper, $\mathrm{n}^{\circ} 20$.

Burman, J.P. (1980), «Seasonal Adjustment by Signal Extraction», Journal of the Royal Statitistical Society, A, 143, 321-337.

Burns, A. F. y Mitchell, W. C. (1946), «Measuring Business Cycles», NBER, New York.

Camacho, M.; Perez-Quiros, G. y Saiz, L. (2006), «Are European business cycles close enough to be just one?», Journal of Economic Dynamics and Control, Elsevier, vol. 30(9-10), 1687-1706.

- (2005), «Do European Business Cycles Look Like One?», Documentos de Trabajo $\mathrm{n}^{\circ} 0518$, Banco de España.

Cancelo, J. R. y Uriz, P. (2001), «A New Approach to Polarization and Conflict. Regime Switching Models for Indicators Derived from Cyclical Chronologies, with an Application to international Cyclical Synchronization», Working papers, Instituto de Estudios Económicos de Galicia.

Cotis, J. P. y Coppel, J. (2005), «Business Cycle Dynamics in OECD Countries: Evidence, Causes and Policy Implications», The Changing Nature of Business Cycles, Reserve Bank of Australia Economic Conference, Sidney, July 2005.

De Lucas, S. (2008), Ciclo y Convergencia: una Perspectiva Mundial, 1950-2006, Tesis Doctoral. Estrella, A. (2007), «Extracting Business Cycle Fluctuations: What Do Time Series Filtres Really Do?», Federal Reserve Bank of New York Staff Reports, n. 289, June 2007

Harding, D. y PAGAN, A. (2002), «Dissecting the cycle: a methological investigation», Journal of Monetary Economics, 49, 365-381.

Hodrick, R. J. y Prescott, E. C. (1980), «Post war U.S. Business Cycles: An Empirical Investigation», Journal of Money, Credit and Banking, 29, 1.

International Monetary Found (2007), «The Changing Dynamics of the Global Business Cycles», World Economic Outlook, Gobalization an Inequality, October 2007

Krolzing, H. M. y Toro, J. (2005), «Classical and Modern Business Cycle Measuremente: The European case», Spanish Economic Review, 7, 1-21.

Maddison, A.(2007), «Historical Statistics for the World Economy: 1-2003AD».

PEÑA, D. (2002), «Análisis de datos multivariantes», MacGraw-Hill Interamericana de España, S.A.U.

Solomou, S. (1998), Economic cycles. Long cycles and business cycles since 1870, Manchester University Press, New York.

Stock, J. y Watson, M. (1989), «New Indexes of Coincident and Leading Economic Indicators», en O. Blanchard y S. Fisher (eds.), NBER Macroeconomic Annual: 1989, Cambridge, MIT Press, 351-394.

- (1999), «Forecasting Inflation», Journal of Monetary Economics, 44, págs. 293-335.

- (2002), «Forecasting Using Principal Components From a Large Number of Predictors», Journal of the American Statistical Association, December 2002, vol. 97, N. 460, Theory and Methods.

- (2003), Introduction to Econometrics, Addison Wesley. 


\section{AneXo 1}

Figura 1. Ciclos comunes de Hodrick-Prescott

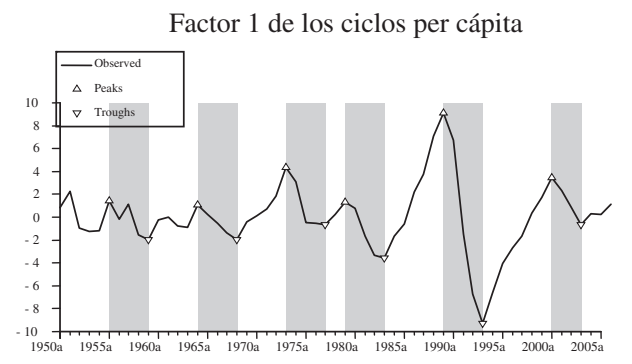

Factor 2 de los ciclos per cápita

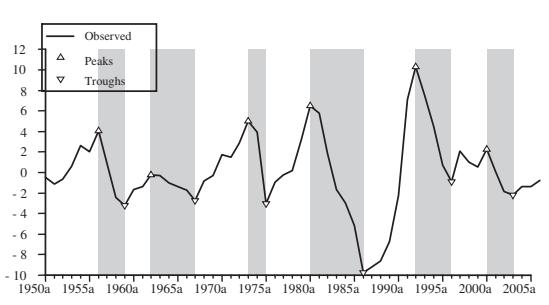

Figura 2. Ciclos comunes de Baxter-King

Factor 1 de los ciclos per cápita

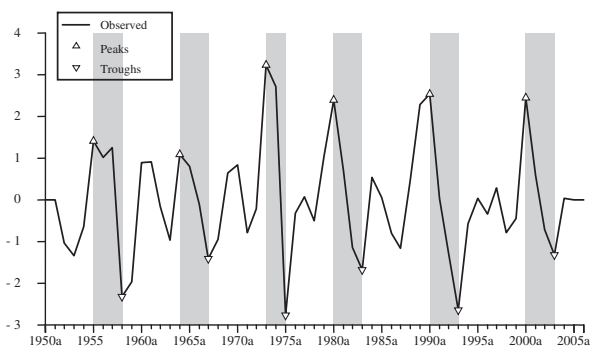

Factor 2 de los ciclos per cápita

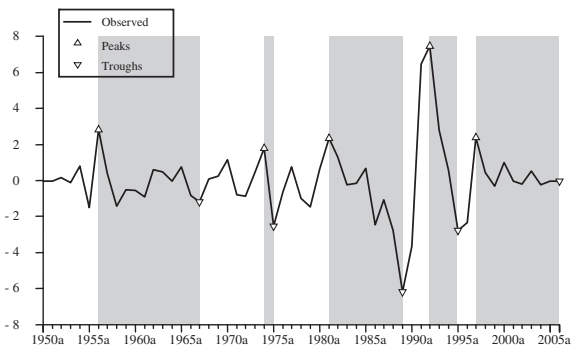

Figura 3. Ciclos comunes de Butterworth

Factor 1 de los ciclos per cápita

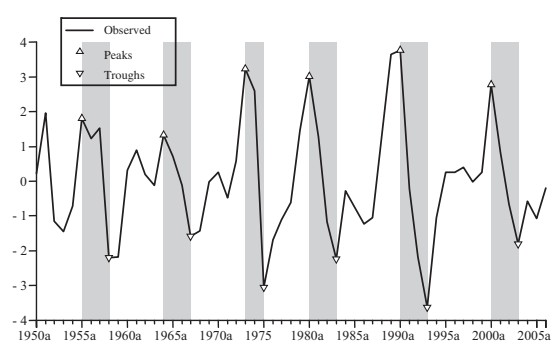

Factor 2 de los ciclos per cápita

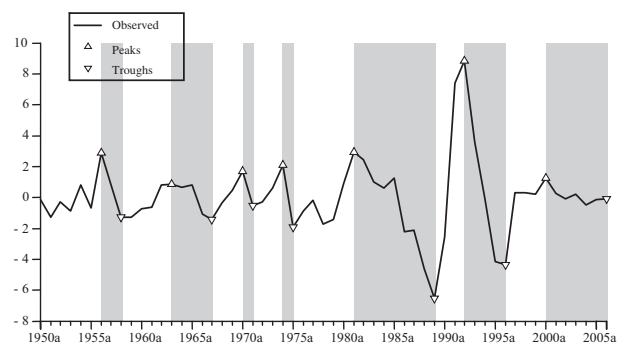

